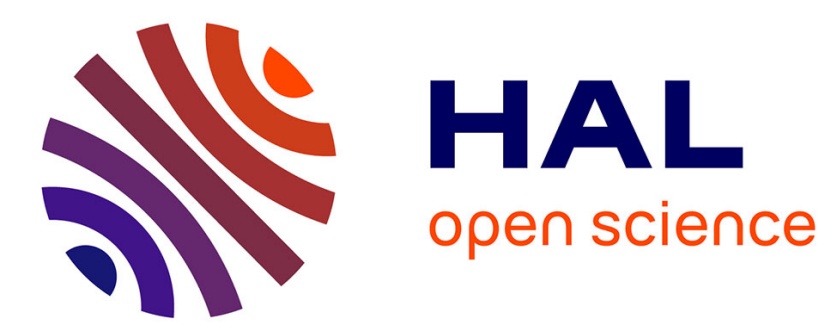

\title{
Brangäne: a new family of Barbarian asteroids
} A Cellino, S Bagnulo, P Tanga, M Devogèle, Ph Bendjoya, E Reilly, J-P

Rivet, F Spoto

\section{To cite this version:}

A Cellino, S Bagnulo, P Tanga, M Devogèle, Ph Bendjoya, et al.. Brangäne: a new family of Barbarian asteroids. Monthly Notices of the Royal Astronomical Society, 2019, 485 (1), pp.570-576. 10.1093/mnras/stz451. hal-03041725

\section{HAL Id: hal-03041725 \\ https://hal.science/hal-03041725}

Submitted on 5 Dec 2020

HAL is a multi-disciplinary open access archive for the deposit and dissemination of scientific research documents, whether they are published or not. The documents may come from teaching and research institutions in France or abroad, or from public or private research centers.
L'archive ouverte pluridisciplinaire HAL, est destinée au dépôt et à la diffusion de documents scientifiques de niveau recherche, publiés ou non, émanant des établissements d'enseignement et de recherche français ou étrangers, des laboratoires publics ou privés. 


\title{
Brangäne: a new family of Barbarian asteroids
}

\author{
A. Cellino ${ }^{1}$, S. Bagnulo ${ }^{2}$, P. Tanga ${ }^{3}$, M. Devogèle ${ }^{4}$, Ph. Bendjoya $^{3}$, E. Reilly $^{2}$, \\ J.-P. Rivet ${ }^{3}$ and F. Spoto ${ }^{3,5}$ \\ ${ }^{1}$ INAF - Osservatorio Astrofisico di Torino, Pino Torinese 10025, Italy. E-mail: alberto.cellino@inaf.it \\ 2 Armagh Observatory, College Hill, Armagh BT61 9DG, UK. \\ 3 Université de la Côte d'Azur, Observatoire de la Côte d'Azur, CNRS, Laboratoire Lagrange, France \\ ${ }^{4}$ Lowell Observatory, 1400 West Mars Hill Road, Flagstaff, AZ 86001, USA \\ ${ }^{5}$ IMCCE, Observatoire de Paris, 77 av. Denfert-Rochereau, 75014 Paris, France
}

Accepted After. Received Before; in original form: Earlier

\begin{abstract}
The so-called Barbarian asteroids (from the name of the prototype of this class, asteroid (234) Barbara) are extremely interesting objects because they might be the survivors of a very early generation of planetesimals. We have identified in the past the first case of an asteroid family (the Watsonia family) whose members are Barbarians, all issued from the collisional disruption of a sizeable parent body. Here we report on the identification of another family, called after the name of its biggest member, asteroid (606) Brangäne, consisting of objects displaying the polarimetric properties characterizing the Barbarian behaviour. This is the first recognized case of a swarm of small Barbarians issued from a quite recent cratering event.
\end{abstract}

Key words: asteroids: polarization - asteroids: composition.

\section{INTRODUCTION}

The discovery of an anomalous polarimetric behaviour of (234) Barbara (Cellino et al. 2006) was the first recognition of the existence of a new class of asteroids (now commonly referred to as the Barbarians) which are very interesting in many respects. Some of these objects are known to exhibit anomalous reflectance spectra, characterized by features that were interpreted by Burbine et al. (1992); Sunshine et al. (2008) as diagnostic of a very high content of refractory compounds on their surfaces, including primarily the aluminous spinel mineral $\left(\mathrm{MgAl}_{2} \mathrm{O}_{4}\right)$. This is a major component of the so-called Calcium Aluminum-rich Inclusions (CAI) found in some meteorites. With their ages around 4.567 Gyrs, derived from isotopic abundances, CAIs are the oldest samples of solid matter found in the Solar system. As pointed out by Sunshine et al. (2008), CAIs are generally classified, on the basis of petrography and geochemistry, into three major groups. Among them, the so-called "fluffy type A" (FTA), characterized by a porous, strongly non-compact structure, is found in all chondritic meteorites, and produces a strong absorption feature in the reflectance spectrum, interpreted as a consequence of a high concentration of FeO-rich spinel. It is also believed that, as opposite to the other types of CAIs, FTAs may never have been totally melted by any transient heating event after their formation.
The most extensive analysis of objects belonging to the Barbarian class, based on both spectroscopic and polarimetric data, has been recently published by Devogèle et al. (2018). These authors found conclusive evidence that all Barbarians, which had been previously classified as members of different taxonomic classes based on their reflectance spectra at visible wavelengths, once the properties of both the visible and near IR regions of the reflectance spectrum are taken into account, appear to belong to a unique taxonomic class: the L class defined by DeMeo et al. (2009).

Devogèle et al. (2018) found that the spectra of L-class objects can be successfully modeled using mixtures of the fluffy kind of spinel present in CAIs, MgO-rich olivine and the mineral compounds found in the matrix of CV3 meteorites. They also found that the presence of CAIs is responsible for the anomalous polarimetric inversion angle characterizing the Barbarians (for an explanation of this and other polarimetric parameters, see, for instance, Cellino et al. 2015). In particular, they pointed out the presence of a variation of the inversion angle of asteroid (234) Barbara as a function of wavelength, and interpreted it as a consequence of the known wavelength-dependent variation of the refractive index of the spinel mineral.

Until a couple of years ago, it was not clear whether the properties of Barbarians were determined by anomalous surface properties, or by structural composition. In this respect, a major step forward has been the discovery by means of polarimetric measurements that the Watsonia asteroid family 
Table 1. Total number of currently recognized members of the Brangäne family, and the lower and upper limits of the intervals covered by its members in the space of the proper orbital elements semi-major axis, eccentricity and sine of inclination.

\begin{tabular}{cccc}
\hline \hline $\begin{array}{c}\text { N. of } \\
\text { members }\end{array}$ & $\begin{array}{c}\text { proper semi-major } \\
\text { axis (AU) }\end{array}$ & $\begin{array}{c}\text { proper } \\
\text { eccentricity }\end{array}$ & $\begin{array}{c}\text { sine of proper } \\
\text { inclination }\end{array}$ \\
\hline 325 & $2.571-2.597$ & $0.178-0.183$ & $0.165-0.168$ \\
\hline
\end{tabular}

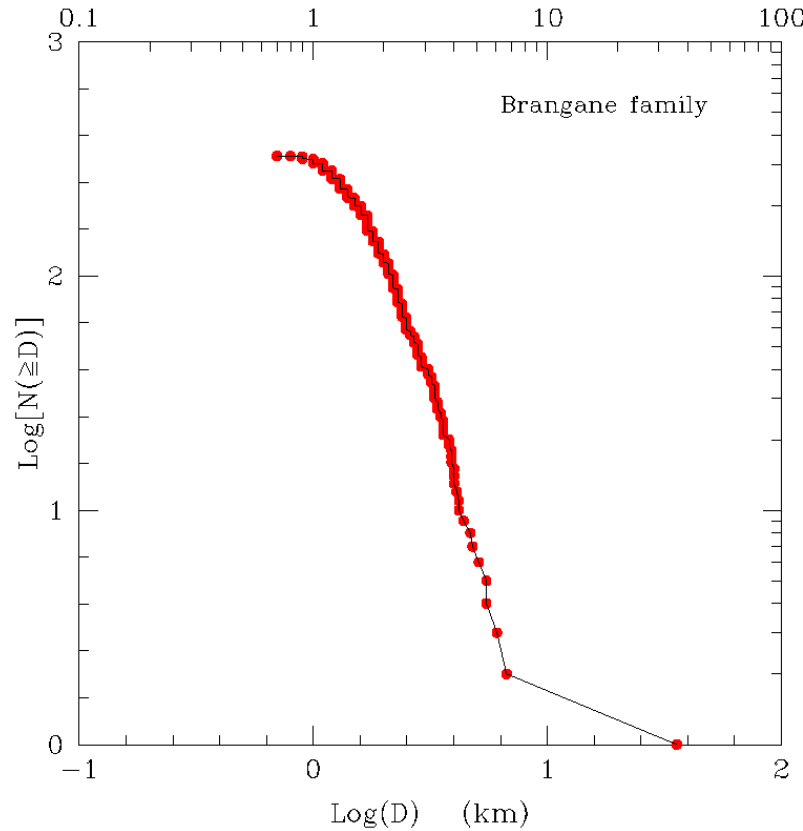

Figure 1. Cumulative size distribution of the Brangäne family. The typical trend characterized by a large difference in size between the largest member and the rest of the family members clearly shows that the family is the outcome of a cratering event. At sizes smaller than about $2 \mathrm{~km}$ the size distribution becomes shallower, an indication of incompleteness of the recognized smallest members

(Novaković et al. 2011) consists of Barbarian objects (Cellino et al. 2014).

Asteroid families are groups of objects issued by the collisional disruption of single parent bodies. The fact that the members of the Watsonia family are Barbarians strongly suggests that the Barbarian properties are not purely surface effects, because the members of a same family derive from material located at different depths inside the parent body. This was also the first discovery of Barbarian asteroids of relatively small sizes.

The main belt asteroid (606) Brangäne, about $40 \mathrm{~km}$ in size (Mainzer at al. 2016), belongs to the L-class (DeMeo et al. 2009). It is the biggest member of another family (Milani et al. 2014). Table 1 summarizes the main parameters of the Brangäne family which, according to Spoto et al. (2015) is the result of a very recent cratering event (the age between 30 and 50 Myrs being derived by the analysis of the "V-shaped" diagram, see below) In this Table, the data

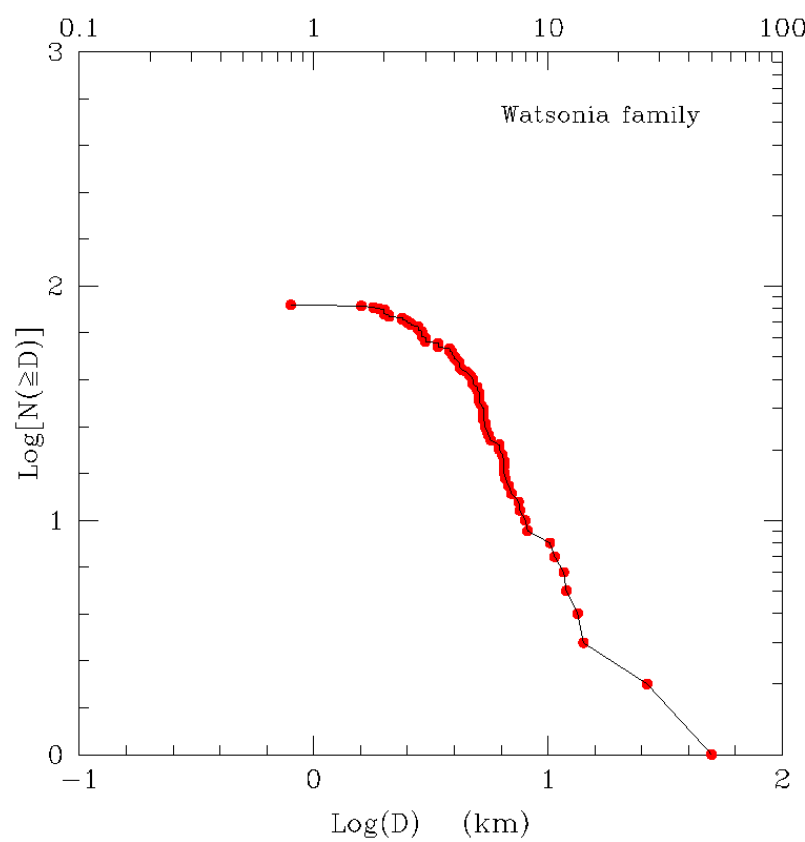

Figure 2. The same as Fig. 1, but for the family of Watsonia, the only one previously identified family of Barbarians (Cellino et al. 2014). As shown by the morphology of its cumulative size distribution, the Watsonia family may suggest a more energetic disruption event, rather than a typical cratering event as in the case of the Brangäne family.

have been taken from the AstDys site ${ }^{1}$, where a regularly updated list of members of asteroid families is publicly available.

The cumulative size distribution of the family is shown in Fig. 1. The sizes of the family members have been taken directly from the NEOWISE catalog (Mainzer at al. 2016), whenever available. The same albedo data have been also used to derive the average albedo of the family, $0.116 \pm$ 0.005 , very similar to the geometric albedo of Brangäne itself $(0.113 \pm 0.021)$. The sizes of family members not observed by WISE were computed from the known relation linking the size to the absolute magnitude $\mathrm{H}$ and the geometric albedo $p_{V}$ :

$\log (D)=3,1236-0.2 H-0.5 \log \left(p_{V}\right)$

where $D$ is the equivalent diameter in $\mathrm{km}$. In the computations, the value of $H$ was taken from the file listing the family members available in the AstDys site. For the value of the geometric albedo $p_{V}$ we adopted the NEOWISE based average value of 0.116 mentioned above.

The Brangäne family is the likely outcome of a cratering event. This is suggested by the large difference in size between its largest member, (606) Brangäne, having an absolute magnitude $H=10.22$ and an estimated size of about $36 \mathrm{~km}$ (Mainzer at al. 2016), and the rest of the family members. The latter have absolute magnitudes generally fainter than 14, and sizes mostly below $5 \mathrm{~km}$. In the cases of families issued from cratering events, the overall trend of the cumulative size distribution tends to exhibit at the largest sizes

1 http://hamilton.dm.unipi.it/astdys/index $\cdot$ php?pc=5 
a characteristic concave trend (Tanga et al. 1999; Durda et al. 2007).

For a comparison, Fig. 2 shows the cumulative size distribution of the Watsonia family. The morphology of the size distribution, computed in exactly the same way as in the case of Brangäne, as explained above, is clearly different with respect to the case of Brangäne, as it is not characterized by a big difference between the size of the largest member and those of the other biggest family members. It can also be noted that in the case of Watsonia there is a stronger depletion of objects at the low-end of the size distribution. This can be partly due to observational bias, due to the fact that the perihelion distances of the Brangane family are lower than those of Watsonia members, due to a non-negligible difference in orbital eccentricity. On the other hand, the two families are located at fairly similar heliocentric distances (the orbital semi-major axis of (729) Watsonia is about 2.76 AU) and, consisting of objects displaying similar spectroscopic and polarimetric properties, also the albedo of the members of the two families should be similar. Our preferred interpretation is that Watsonia represents the outcome of a collision producing a more extensive disruption of the parent body, and the relative lack of small family members with respect to the case of the Brangäne family could be diagnostic of an older age, because the progressive depletion of the smallest members of a family due to the Yarkovsky effect increases with time (Rubincam 1995; Farinella et al. 1998). This interpretation is also supported by the apparently shallower slope of the cumulative size distribution of the Watsonia family if we compare it with that of the Brangäne family. A progressive decrease of slope as a function of time is expected to be a typical result of a Yarkovsky-driven erosion.

The reason why the Brangäne family is so interesting is that we recently discovered that (606) Brangäne itself is a Barbarian (Devogèle et al. 2018). It was therefore very tempting to carry out a campaign of polarimetric observations to check whether we could find a new example of a family consisting of Barbarian objects. The results of this investigation are presented in this paper.

\section{NEW OBSERVATIONS}

Our target list included ten objects that are members of the Brangäne family according to the list of family members available at the AstDys web site. These objects are listed in Table 2 .

The light that we receive at visible wavelengths from the asteroids is scattered sunlight, and is in a state of partial linear polarization. The plane of linear polarization is always found to be either parallel or perpendicular to the Sun - target - observer plane (scattering plane). Usually, in the literature the state of linear polarization is described by the so-called $P_{\mathrm{r}}$ parameter, which is equal in module to the fraction $P$ of linear polarization. The difference between $P_{\mathrm{r}}$ and $P$ is that $P_{\mathrm{r}}$ is expressed with a sign that is positive if the plane of linear polarization is perpendicular to the scattering plane, whereas it is negative if the plane of linear polarization is par-

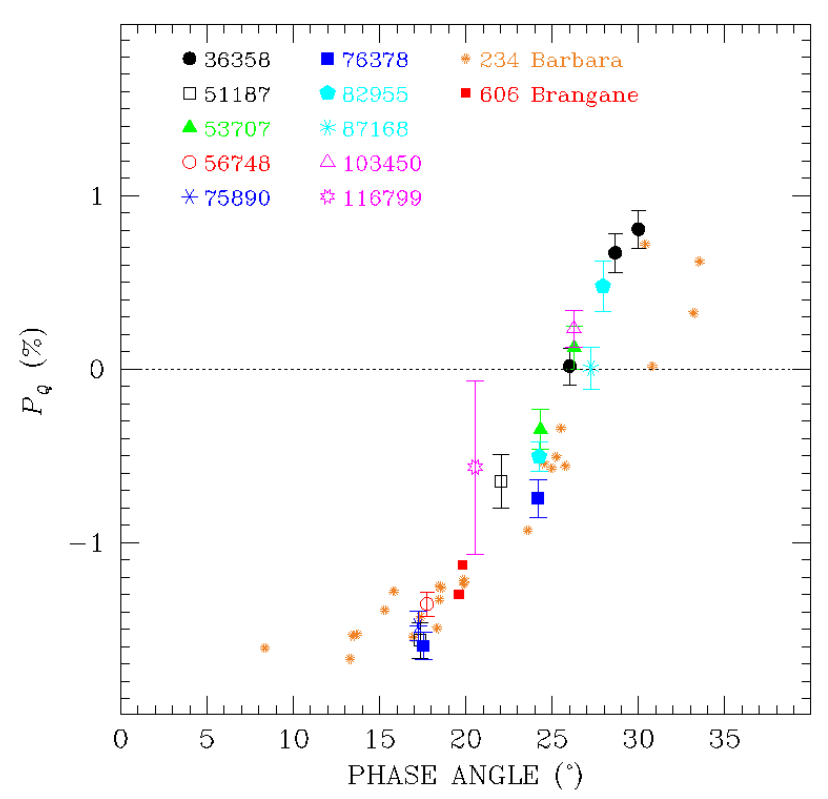

Figure 3. Polarization versus phase-angle data for the targets of the present investigations, as well as a couple of measurements of (606) Brangäne, the largest member of the family, previously observed at the Calern observatory. The family data are compared with the polarization curve of the prototype of Barbarians, (234) Barbara, whose available measurements are shown, for sake of clarity, without their associated error bars (orange symbols).

allel to the scattering plane. At small phase angles $\left(\ll 20^{\circ}\right)$, all asteroids exhibit the phenomenon of socalled negative polarization, characterized by $P_{\mathrm{r}}<0$. Conversely, at phase angles $\gtrsim 20^{\circ}$ nearly all asteroids exhibit positive polarization. By contrast, at phase angle $\simeq 20^{\circ}$, Barbarians still exhibit a relatively high fraction of negative polarization $\left(P_{\mathrm{r}} \sim-1 \%\right)$. Barbarian polarization becomes positive only at phase-angle $\gtrsim 27 \div 30^{\circ}$. Therefore, to identify Barbarians, we decided to measure the polarization at phase angles around $17^{\circ}$ or slightly larger, and establish whether the measured polarization plane is found to be parallel or perpendicular to the scattering plane.

We observed our targets using the VLT FORS2 instrument (Appenzeller et al. 1998) in imaging polarimetric mode, and obtained 15 broadband linear polarization measurements in the $R$ special filter from April to September 2017. Polarimetric measurements were performed with the retarder wave-plate at all positions between $0^{\circ}$ and $157.5^{\circ}$, at $22.5^{\circ}$ steps. For each observation, the exposure time accumulated over all exposures varied from $720 \mathrm{~s}$ (for 87168) to $2857 \mathrm{~s}$ (for 76378 ). Polarimetric data were then treated as explained in Bagnulo et al. (2016), and, consequently, reduced Stokes parameters $P_{Q}=Q / I$ and $P_{U}=U / I$ were calculated adopting as a reference direction the perpendicular to the great circle passing through the object and the Sun. This way, $P_{Q}$ represents the flux perpendicular to the scattering plane minus the flux parallel to that plane, divided by the sum of these fluxes, and is therefore by definition identical to $P_{\mathrm{r}}$ ). For symmetry reasons, $P_{U}$ values are expected to be zero, and inspection of their values allows us to perform an indirect quality check of the $P_{Q}$ 
Table 2. Polarimetry and photometry of ten asteroids of in the special $R$ filter. $P_{Q}$ and $P_{U}$ are the reduced Stokes parameters measured in a reference system such that $P_{Q}$ is the flux perpendicular to the plane Sun-Object-Earth (the scattering plane) minus the flux parallel to that plane, divided by the sum of the two fluxes. In literature $P_{Q}$ is usually designated with the symbol $P_{\mathrm{r}}$. In the last columns, $m_{R}(\alpha)$ and $m(\alpha)$ are the magnitude reduced to unit distance from the observer and from the Sun, and the apparent magnitude, respectively.

\begin{tabular}{|c|c|c|c|c|c|c|c|c|}
\hline $\begin{array}{c}\text { Date } \\
\text { (yyyy mm dd) }\end{array}$ & $\begin{array}{l}\text { Time (UT) } \\
\text { (hh:mm) }\end{array}$ & $\begin{array}{l}\text { Exp } \\
(\mathrm{sec})\end{array}$ & Object & $\begin{array}{c}\text { Phase angle } \\
\text { (DEG) }\end{array}$ & $\begin{array}{l}P_{Q} \\
(\%)\end{array}$ & $\begin{array}{l}P_{U} \\
(\%)\end{array}$ & $m_{R}(\alpha)$ & $m(\alpha)$ \\
\hline 2017-07-04 & 09:53 & 904 & 36358 & 30.00 & $0.82 \pm 0.11$ & $0.03 \pm 0.11$ & $16.07 \pm 0.20$ & $18.88 \pm 0.20$ \\
\hline 2017-08-01 & $06: 16$ & 904 & & 28.66 & $0.67 \pm 0.11$ & $0.06 \pm 0.11$ & $16.22 \pm 0.21$ & $18.71 \pm 0.21$ \\
\hline $2017-08-17$ & 09:29 & 496 & & 26.02 & $0.02 \pm 0.11$ & $0.03 \pm 0.11$ & $16.24 \pm 0.20$ & $18.53 \pm 0.20$ \\
\hline 2017-04-26 & 09:28 & 824 & 51187 & 22.04 & $-0.84 \pm 0.17$ & $0.13 \pm 0.17$ & $20.25 \pm 0.13$ & $22.98 \pm 0.13$ \\
\hline $2017-07-28$ & 02:01 & 860 & & 17.34 & $-1.56 \pm 0.10$ & $0.06 \pm 0.10$ & $18.48 \pm 0.52$ & $21.31 \pm 0.52$ \\
\hline 2017-06-19 & 01:14 & 992 & 53707 & 24.33 & $-0.35 \pm 0.12$ & $0.18 \pm 0.12$ & $16.08 \pm 0.16$ & $19.23 \pm 0.16$ \\
\hline $2017-07-24$ & $23: 45$ & 1568 & & 26.29 & $0.12 \pm 0.13$ & $0.02 \pm 0.13$ & $16.01 \pm 0.19$ & $19.48 \pm 0.19$ \\
\hline 2017-09-20 & 04:40 & 1568 & 56748 & 17.76 & $-1.36 \pm 0.07$ & $0.17 \pm 0.07$ & $16.38 \pm 0.28$ & $19.00 \pm 0.28$ \\
\hline $2017-06-25$ & 01:01 & 1496 & 75890 & 17.24 & $-1.48 \pm 0.08$ & $-0.01 \pm 0.08$ & $16.40 \pm 0.12$ & $19.14 \pm 0.12$ \\
\hline $2017-07-17$ & $02: 36$ & 2778 & & 22.25 & $-0.79 \pm 0.14$ & $-0.06 \pm 0.14$ & $16.41 \pm 0.11$ & $19.49 \pm 0.11$ \\
\hline $2017-06-21$ & 04:34 & 1368 & 76378 & 17.54 & $-1.60 \pm 0.08$ & $-0.08 \pm 0.08$ & $16.31 \pm 0.11$ & $18.69 \pm 0.11$ \\
\hline $2017-07-17$ & 03:33 & 2856 & & 24.18 & $-0.75 \pm 0.11$ & $0.20 \pm 0.11$ & $16.73 \pm 0.28$ & $19.50 \pm 0.11$ \\
\hline $2017-06-23$ & $00: 30$ & 1136 & 82955 & 24.25 & $-0.50 \pm 0.08$ & $0.05 \pm 0.08$ & $16.30 \pm 0.11$ & $18.86 \pm 0.11$ \\
\hline $2017-08-16$ & 01:02 & 2376 & & 27.95 & $0.48 \pm 0.14$ & $0.48 \pm 0.14$ & $16.43 \pm 0.13$ & $19.62 \pm 0.13$ \\
\hline $2017-08-28$ & $09: 26$ & 720 & 87168 & 27.26 & $-0.08 \pm 0.12$ & $0.07 \pm 0.12$ & $16.78 \pm 0.16$ & $18.97 \pm 0.16$ \\
\hline 2017-06-22 & $23: 46$ & 2072 & 103450 & 26.26 & $0.23 \pm 0.11$ & $0.13 \pm 0.11$ & $16.53 \pm 0.19$ & $19.74 \pm 0.19$ \\
\hline 2017-09-12 & $03: 23$ & 2376 & 116799 & 20.55 & $-0.86 \pm 0.15$ & $0.06 \pm 0.15$ & $16.61 \pm 0.06$ & $19.48 \pm 0.06$ \\
\hline
\end{tabular}

values. Polarimetric measurements are given in cols. 6 and 7 of Table 2 .

Each polarimetric series was preceded by the acquisition of an image to fine-tune the telescope pointing and ensure target is properly centred and not vignetted by the Wollaston masks. These acquisition images were used for photometric measurements. PSF fitting photometry of all objects in the images was performed using the procedures in the DAOPHOT package of iraf using the following strategy. Objects where a maximum of ten iterations were required in order to fit the PSF and no error was recorded for the resulting photometry were kept for the following steps. The objects were matched by sky coordinate to those recorded in the PanSTARRS Mean Object Catalog. For each image, a linear regression model was fit for the measured apparent magnitude $m_{r}$, to the Mean PSF magnitude, $m_{r}$ (PanSTARRS), recorded by PanSTARRS in the $r$ filter. An average of 100 objects per image were used to generate the model. The model was then used to predict the magnitude of the target asteroid. Reduced magnitudes were computed using helio- and geo-centric distances taken from HORIZONS (Giorgini et al. 1996), and are given in Table 2, together with the corresponding apparent magnitudes. It is important to comment that, at least in the present case, we do not expect that individual photometric measurements can provide the same physical insight as polarimetric measurements, as the limited phase-angle range covered by our observations does not even allow us to estimate the slope of the magnitude-phase angle curve.
However, both photometry and polarimetry data could be useful in a future work for modelling purposes, if more data are collected.

It is important to note also that the errors on the polarimetric measurements were calculated based only on photon noise using normal error propagation of the flux errors (see, e.g., Bagnulo et al. 2009). Systematics with FORS2 are of the order of a few units in $10^{-4}$ (e.g., Bagnulo et al. 2016). It is also useful to point out that FORS acquired polarimetric standard stars within its calibration plan, and the fact that $P_{U}$ is always consistent with zero, means that the polarimetric optics were consistently well aligned.

Our polarimetric measurements are shown in Figure 3, in which we show also two measurements of (606) Brangäne obtained in the $V$ filter by Devogèle et al. (2018). A comparison is also made with the phase-polarization curve of (234) Barbara, the prototype of this class of objects using literature data (Cellino et al. 2016; Devogèle et al. 2018). and adding the three new measurements of Table 3 obtained in the framework of a recent spectro-polarimetric survey of asteroids (observations and data reduction were performed in the same way as Bagnulo et al. 2015). 
Table 3. New polarimetric measurements of (234) Barbara in the $V$ filter. Uncertainties are somewhat arbitrarily estimated based on our knowledge of the instrument polarization. Contribution from photon-noise is $\lesssim 0.01 \%$.

\begin{tabular}{ccr}
\hline \hline Observation date & $\begin{array}{c}\text { Phase angle } \\
(\mathrm{deg})\end{array}$ & \multicolumn{1}{c}{$\begin{array}{c}P_{Q} \\
(\%)\end{array}$} \\
\hline $2015-05-09$ & 13.5 & $-1.54 \pm 0.05$ \\
$2015-05-27$ & 18.5 & $-1.33 \pm 0.05$ \\
$2016-07-07$ & 33.2 & $0.32 \pm 0.05$ \\
\hline
\end{tabular}

\section{DISCUSSION AND CONCLUSIONS}

All asteroids of our target list exhibit the Barbarian polarimetric behaviour, characterized by a wide negative polarization branch, and an inversion angle which in this case is $\sim 25^{\circ}$. The two independent CAPS (Calern Asteroid Polarimetric Survey) measurements of (606) Brangäne perfectly fit the trend exhibited by the other family members.

A more detailed analysis of Fig. 3 shows that the Brangäne family members very closely mimic the behaviour of (234) Barbara in an interval of phase angles $\sim 17^{\circ} \div \sim$ $25^{\circ}$. At the highest values of phase angle, however, we note that the behaviour of (234) Barbara is not clearly defined because the four measurements at phase-angle $\gtrsim 30^{\circ}$ are not in a good agreement among each other. In particular, the measurement obtained at a phase of $30.4^{\circ}$ obtained by Cellino et al. (2016) fits very well the data obtained for the members of the Brangäne family, but is high when compared with the other measurements obtained at similar or near phase angles, which suggest a much gentler increase of polarization in the positive branch. More observations are needed to better characterize the behaviour of (234) Barbara around phase angle $30^{\circ}$. Based on the observations presently available, the inversion angle could be from $25^{\circ}$ (very similar to the inversion angle for the Brangäne family) to about $30^{\circ}$.

In any case it is evident that our measurements of a sample of members of the Brangäne family definitively show that this is a family of Barbarians, and the observed behaviour of its members suggest an inversion angle of polarization between $25^{\circ}$ and $27^{\circ}$. Interestingly enough, according to Devogèle et al. (2018), such a value would suggest a relatively low abundance of CAI abundances, possibly smaller than $5 \%$. Moreover, although our data do not allow us to derive a robust estimate of the extreme value of negative polarization $P_{\min }$, the visible trend seems to suggest a fairly deep value for this parameter. According to Devogèle et al. (2018) this would also correspond to small abundances of nano-phase iron particles on the surface (see Fig. 13 of Devogèle et al. 2018). Further spectroscopic data in the visible and near-IR spectral regions will help to confirm or rule out these tentative inferences.

The existence of a variety of values for the inversion angle of polarization of different Barbarians can be important, and may correspond to significant differences in terms of surface properties and composition, according to preliminarily inferences by Devogèle et al. (2018). This means that a possible difference of about five degrees between the inversion angle of the Brangäne family and (234) Barbara suggests that the small population of objects that we call Barbar- ians might be more heterogeneous than currently believed. As shown by Devogèle et al. (2018), the reflectance spectra of different Barbarians may also exhibit differences that in some cases might represent more than subtle nuances among objects sharing a given set of properties. In other words, the data currently available suggest that the Barbarians might include objects having non-negligible differences in terms of surface properties and composition. Only by obtaining new data we can confirm or rule out this possibility.

Devogèle et al. (2018) claimed that the polarimetric properties of Barbarians are a consequence of the high content of spinel-bearing minerals in their surfaces, confirming previous results by Sunshine et al. (2008). This may suggest a formation dating back to epochs when the solid matter accreted into growing planetesimals was characterized by a much higher abundance of refractory components, the first compounds to solidify during the very early cooling of a hot proto-planetary disk. Barbarians could therefore be very old, having possibly survived the very chaotic early phases of evolution of our planetary system.

According to the so-called "Grand Tack" model (Walsh et al. 2011, 2012), the very early history of the Solar system was characterized by episodes of back and forth migration of the giant planets, producing a huge depletion (by more than $99 \%$ ) of the planetesimals originally accreted in the region of the current main asteroid belt. The subsequent evolution, up to the end of the so-called Late Heavy Bombardment phase, about 3.9 Gyrs ago, is described by the so-called "Nice" Model (Gomes et al. 2005; Morbidelli et al. 2005). It is believed that this was also an era of instability for the small bodies, subject to perturbations that led many objects to move from the outer regions to smaller heliocentric distances. At the end of this era, the major planets achieved their current configuration, and the populations of small bodies orbiting at different heliocentric distances started to be more stable, their further evolution being mainly determined by mutual collisions and, in the current asteroid belt, by a steady removal of the smallest objects due to a progressive drift of their orbital semi-major axes determined by thermal emission mechanisms (Yarkovsky effect, see, for instance, Rubincam (1995); Farinella et al. (1998)). So, the lucky survivors of an earlier age are expected to be rare, and larger than a few tens of $\mathrm{km}$.

This could explain why Barbarians are so rare and relatively big: apart from the members of the Watsonia and Brangäne families, there are currently only 17 identified Barbarians in the whole main belt, and all of them are above 40$50 \mathrm{~km}$ in size. Smaller objects could have avoided detection so far simply because they are fainter, but if they were common it is unlikely that a large number of them would not have been identified in spectroscopic surveys like the one carried out by DeMeo et al. (2009), taking into account that the $L$ taxonomic class is characterized by some evident features in the near-IR region of the reflectance spectrum.

Among the few known Barbarians, moreover, there are reasons to believe that four objects, namely (387) Aquitania, (729) Watsonia and (980) Anacostia, as suggested by Cellino et al. (2014), and also (599) Luisa, found more recently to be another nearby Barbarian, could be the surviving members of a previous, first-generation family produced by the collisional disruption of a much bigger Barbarian parent body, 
occurred presumably in very ancient times. The idea is that the parent body of the Watsonia family was likely a fragment from an earlier, first-generation collision involving a much bigger parent body. According to Cellino et al. (2014), this event produced, in addition to the parent body of the Watsonia family, also a few other big Barbarian asteroids (including (387) Aquitania and (980) Anacostia, see also Masiero \& Cellino (2009)). After a very long time since their origin, these objects have orbits still similar to each other and to those of the Watsonia family, but not sufficiently close to each other and to Watsonia to form an identifiable, unique family. In other words, according to Cellino et al. (2014) we would have here the first recognized example of an eroded and dispersed family that was formed during or just later than the early, chaotic phases of the solar system history, characterized by strong perturbations due to processes of migration of the giant planets. The few survivors of this very old family are still recognizable today as siblings sharing a same origin, only as a consequence of their peculiar physical properties.

The age of this first-generation family that supposedly produced (729) Watsonia and the big Barbarian asteroids nearby, must be older than the upper limit found by Spoto et al. (2015) for the ages of families still recognizable today using current techniques of family identification and dating. The techniques of family identification are purely based on the vicinity in the proper elements space, while the ages are derived from the measurement of a size-dependent spreading in proper semi-major axis of family members due to the Yarkovsky effect, derived from analysis of the so-called "Vshaped" diagrams of families ${ }^{2}$. In other words, the ages are estimated by means of an analysis of the time needed to produce the observed, size-dependent spreading in orbital semi-major axis of the family members, as a consequence of the Yarkovsky effect (Spoto et al. 2015; Milani et al. 2017). In the case of the Watsonia family, an estimate of the age is uncertain, due to the asymmetric structure of the family. The age turns out to range between about 0.8 and 1.2 Gyrs, depending on the unknown density of its members. Such an age, in spite of the large uncertainty, supports the assumption that the supposedly big primordial family that produced the Watsonia parent body could have an age of the order of, if not larger than, $2 \cdot 10^{9}$ years, older than any other known family.

If the few existing Barbarians (apart from the small members of more recent families) are long-sought survivors from a very early epoch, we might be close to have found some pieces of the Holy Grail in asteroid science. In this respect, many primitive, low-albedo asteroids that are abundant in the current asteroid main belt are thought to have been originally accreted at much higher heliocentric distances (Gomes et al. 2005; Morbidelli et al. 2005). Recently, Delbò et al. (2017) found indications of the existence in the inner asteroid belt of low-albedo objects that might be the survivors of at least one asteroid family possibly originated as early as 4 billion years ago. It is not clear whether the supposed parent body of this family can be representative

2 Plots of the V-shaped diagrams of asteroid families can be displayed using the Plotting the asteroids facility available at http://hamilton.dm.unipi.it/astdys2/Plot/ of a population of dark planetesimals accreted at relatively small heliocentric distances, or might have been a member of a migrating population of planetesimals originally accreted at larger heliocentric distances.

However, the Barbarians look different with respect to low-albedo objects, whatever their origin might be. In particular, Barbarians seem to have significantly higher albedos (mostly between about 0.15 and 0.20 , according to Cellino et al. 2016), and are found in the current asteroid main belt in a wide interval of heliocentric distances.

Devogèle et al. (2018) found that the $2.5 \mu \mathrm{m}$ absorption band which characterizes the reflectance spectra of Barbarians seems to be diagnostic of the presence of iron-enriched spinel mineral present in CAIs. In particular, they proposed that Barbarians are characterized by a composition rich in fluffy-type CAIs. The production of this mineral assemblage seems to require a mild, but not negligible degree of thermal alteration, whereas a strong thermal alteration would be expected to destroy the fluffy-type, iron-enriched variety of spinel. The interior of planetesimals big enough to include a significantly large amount of $\mathrm{Al}_{26}$ seems not to be a favorable environment. In this respect, the existence of a big Barbarian parent body from which a first generation of fragments including (729) Watsonia and its nearby Barbarian siblings (Aquitania, Anacostia, etc.) originated can be a problem, since the required size of such parent body cannot be less than $200 \mathrm{~km}$. Of course, more detailed models of the internal structure and evolution of such a body is needed to confirm or rule out the compatibility with the presence and survival up to our days of large amounts of fluffy-type, spinel rich CAI materials.

In general terms, a composition enriched in refractory minerals can be more compatible with an origin in a hot environment at moderate heliocentric distances, because in a colder environment a larger variety of minerals can exist in the solid state, making it more difficult to explain a strong enrichment in refractory compounds.

The determination of the most likely astrophysical environment to explain the origin of Barbarians is an open question, due to our insufficient understanding of the composition of these objects. Devogèle et al. (2018) obtained reasonable fits of the observed spectroscopic properties of Barbarians by considering a mineral assemblage composed by a mixture of fluffy CAI, MgO-rich olivine and minerals present in the matrix of a couple of CV3 meteorites. The CV3 are low-albedo, primitive meteorites, and for some of them the presence of aqueous alteration cannot be ruled out, something that is in tension with an origin in a hot environment. Mg-rich silicates, however, are predicted by thermodynamical models to condense in a hot gas at temperature above $1200 \mathrm{~K}$, whereas the reaction with Fe occurs at lower temperatures. According to Hanner and Bradley (2004), Mg-rich olivine (forsterite) and pyroxene (enstatite) may have formed by direct condensation in the inner primordial nebula, although both forsterite and enstatite have been found by the Giotto and Vega missions to be present in comet 1P/Halley (Schultze et al. 1997), and, more recently, in comets Hale-Bopp (Crovisier et al. 1997) and 9P/Tempel (Lisse et al. 2007), based on thermal IR spectra. They have been also directly found in samples of comet $81 \mathrm{P} / \mathrm{Wild} 2 \mathrm{col}-$ lected by the Stardust mission (Clemett et al. 2007).

All this suggests that we cannot rule out the possibility 
that Barbarians may be the few survivors of a pristine population of moderate-sized, early planetesimals accreted at the beginning of planetary formation at moderate heliocentric distances. The situation, however, is still strongly ambiguous and we have not yet a sufficient body of evidence to allow us to draw firm conclusions about the origin of these bodies, and we cannot yet discriminate among very different, possible scenarios.

The identification of a new family of Barbarians is in any case an important result. The total number of identified Barbarians increases by many tens of objects, if we assume that, apart from some fraction of random interlopers (Migliorini et al. 1995), most members of the Brangäne family, including those that have not yet been observed, are Barbarians. On the other hand, all the members of any given family (apart from possible special cases of mutuallyoverlapping multi-generation events) are fragments produced by the disruption of one single parent body. This means that all the members of a given family normally correspond to one single asteroid of a previous generation. The Watsonia and Brangäne family members are fragments of two, larger Barbarians that were disrupted in relatively recent times. In particular, there is evidence that the Brangäne family is very young Spoto et al. (2015).

It is worth noting that the two impacts that produced the Brangane and Watsonia families did not cause a widespread alteration of the polarimetric properties of their members, despite the fact that one was a catastrophic event and the other a cratering (which would create fresh-looking ejecta but leave a largest remnant having on the average a much older surface). This may imply that the long inversion angle property that characterizes Barbarians is not affected by compaction, weathering, regolith size, or other time-dependent effects, in agreement with results of other studies. The recent production of small Barbarian fragments in the asteroid main belt is interesting, because it means that at least some of these objects can have chaotically evolved, being subject to the Yarkovsky effect, up to possibly achieving near-Earth asteroid-like orbits. As a consequence, it is possible that at least a few Barbarians might exist today among the NEA population. This is exciting, because the identification of some Barbarian NEAs would possibly permit to conceive space missions of exploration and sample-return, making it possible to retrieve to the Earth some materials which might date back to the very early epochs of planetary growth.

In most general terms, the number of existing families of Barbarians and the sizes of their parent bodies put critical constraints on the interpretation of the physical properties and the possible origin of these objects (Devogèle et al. 2018). In particular, the number of Barbarian families may put some constraints on the initial inventory of these bodies, having been subject, since the end of the era of Late Heavy Bombardment, to the general process of collisional evolution of the asteroid population, that can be investigated by means of numerical models (Bottke et al. 2015).

There are many reasons to conclude that the study of the Barbarian asteroids is an important topic in modern planetary science, and can shed some light on the processes of early formation and subsequent evolution of planetesimals in our solar system. Further investigations are needed to solve some of the open questions concerning these interesting objects. This is also another example of the importance of polarimetric studies of asteroids. This discipline has been somehow underexploited for a long time, until recent years that have seen not only the discovery of Barbarians, but also the possibility to use polarimetric properties to infer information about possible links between asteroids and comets, as recently demonstrated in the case of polarimetric observations of (101955) Bennu (Cellino et al. 2018).

\section{ACKNOWLEDGMENTS}

This survey is based on observations collected at the European Organisation for Astronomical Research in the Southern Hemisphere under ESO programme 099.C-0206 $(\mathrm{PI}=$ Cellino). New polarimetric measurements of asteroid (234) Barbara were obtained under ESO programmes 095.C0925 and 097.C-0853 (PI=Bagnulo). New polarimetric measurements of asteroid (606) Brangäne were obtained using the Torino Polarimeter attached to the Omicron West 1-m telescope of the Calern observing station, in the framework of the Calern Asteroid Polarimetric Survey (CAPS) carried out in collaboration between the INAF - Torino Astrophysical Observatory and the Observatoire de la Côte d'Azur (Nice, France).

\section{REFERENCES}

Appenzeller I. et al., 1998, The Messenger, 94, 1

Bagnulo S. et al., 2016, A\&A, 585, A122

Bagnulo, S., Cellino, A., Sterzik, M.J., 2015, MNRAS, 446, L11

Bagnulo S., Belskaya I. N., Boehnhardt H., Kolokolova L., Muinonen K., Sterzik M., Tozzi G.-P., 2011, JQSRT, 112, 2059

Bagnulo S. et al., 2009, PASP, 121, 993

Bottke W. F. et al., 2015, in Asteroids IV (P.Michel, F. E. DeMeo, W. F. Bottke, Eds.), 895, Univ. of Arizona Press, Tucson

Burbine T. H., Gaffey M. J., Bell J. F., 1992, Metic, 27, 424

Cellino A., Bagnulo S., Belskaya I.N., Christou A., 2018, MNRAS Letters 481, L49

Cellino A. et al., 2014, MNRAS 455, 2091

Cellino A., Belskaya I. N., Gil-Hutton R., 2015, Polarimetry of Stars and Planetary Systems (L. Kolokolova, J. Hought, A.-C. Levasseur Regourd, Eds.), 360

Cellino A. et al., 2014, MNRAS Letters, 439, L75

Cellino A., Belskaya I. N., Bendjoya P., di Martino M., GilHutton R., Muinonen K., Tedesco E. F., 2006, Icarus, 180, 565

Clemett S.J., Nakamura-Messenger K., McKay D. S., Sandford S. A., 2007, Lunar and Planet. Inst. Technical Report 38,2091

Crovisier J. et al., 1997, Science 275, 1904

Delbò M., Walsh, K., Bolin, B., Avdellidou, C., Morbidelli, A., 2017, Science, 357, 1026

DeMeo F. E., Binzel R. P., Slivan S. M. and Bus S. J., 2009, Icarus, 202, 160

Devogèle M., et al., 2018, Icarus, 304, 31 
Durda D. D. et al., 2007, Icarus, 186, 498

Farinella P. et al., 1998, Icarus, 132, 378

Giorgini P. et al., 1996, Bulletin of the American Astronomical Society, Vol 28, No. 3, p. 1158

Gomes R. et al., 2005, Nature, 435, 466

Hanner M. S. and Bradley J. P., 2004, Comets II, M. C. Festou, H. U. Keller, H. A. Weaver (eds.), University of Arizona Press, Tucson, p.555-564

Lisse C. M., et al., 2007, Icarus 187, 69

Mainzer A. K. et al., NEOWISE Diameters and Albedos V1.0. EAR-A-COMPIL-5-NEOWISEDIAM-V1.0. NASA Planetary Data System, 2016.

Masiero J., Cellino A., 2009, Icarus, 199, 333

Migliorini F., Zappalà V., Vio R., Cellino A., 1995, Icarus, 118,271

Milani A. et al., 2017, Icarus, 288, 240

Milani A. et al., 2014, Icarus, 239, 46

Morbidelli A. et al., 2005, Nature, 435, 462

Novaković B., Cellino A., Knežević Z., 2011, Icarus, 216, 69

Rubincam D. P., 1995, J. Geophysical Res., 103, 1725

Schultze H., Kissel J., Jessberger E. K., 1997, Astronon. Soc. of the Pacific Conf. Series 122, 397

Spoto F., Milani A., Knežević Z., 2015 Icarus, 257, 275

Sunshine J. M., Connolly H. C., McCoy T. J., Bus S. J., La Croix L. M., 2008, Science, 320, 514

Tanga P., Cellino A., Michel P., Zappalà V., Paolicchi P., Dell'Oro A., 1999 Icarus, 141, 65

Walsh K. J. et al., 2012, Meteoritics \& Planet. Sci., 47, 1941

Walsh K. J. et al., 2011, Nature, 475, 206 